\title{
La Storia contemporanea. Una prospettiva
}

\author{
di Fulvio Cammarano
}

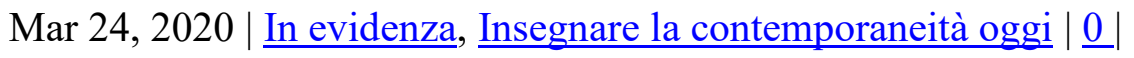

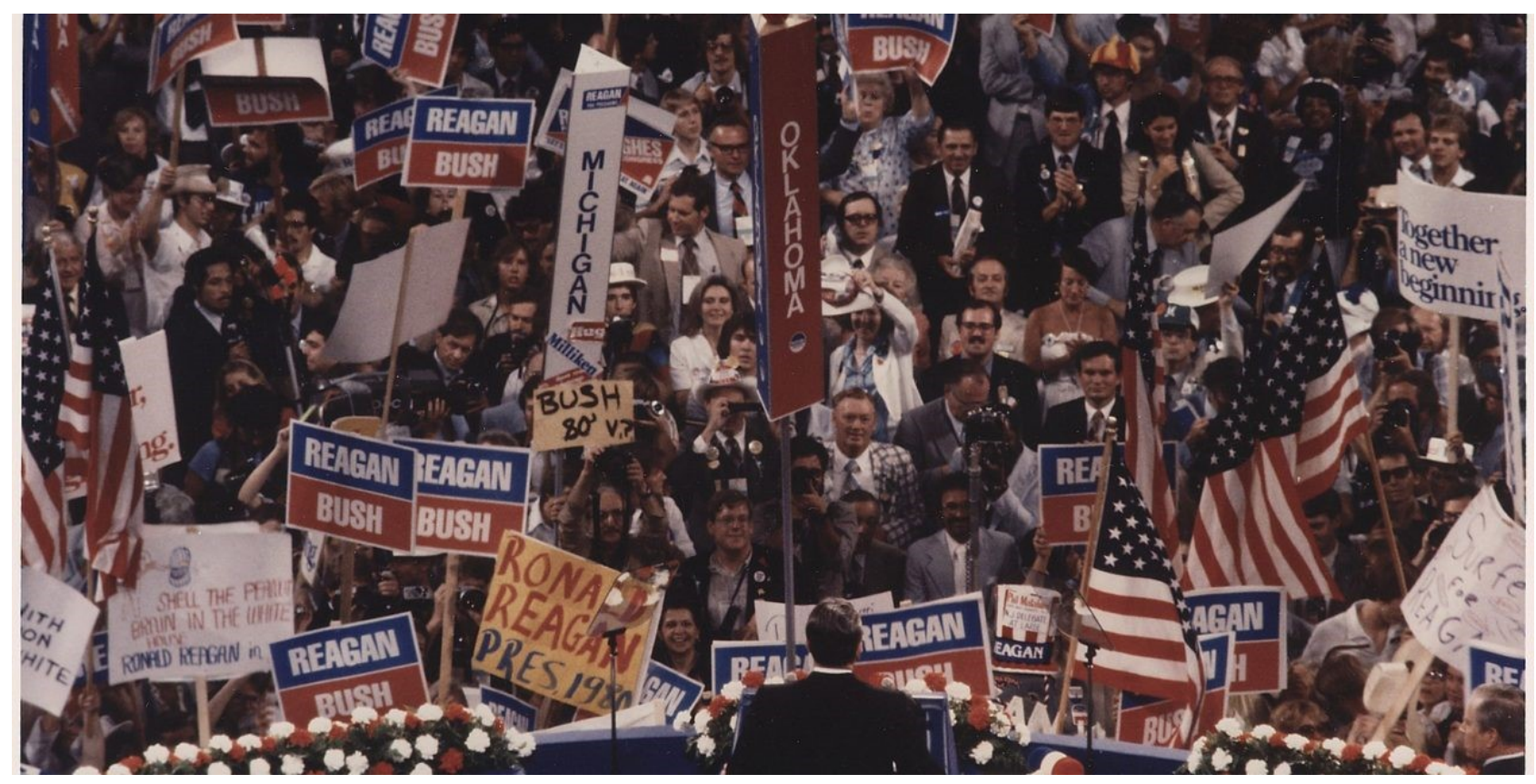

La campagna presidenziale di Ronald Reagan nel 1980

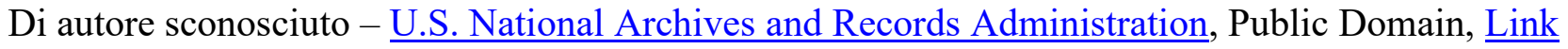

\begin{abstract}
La storia contemporanea, dal punto di vista politico e sociale, inizia con le rivoluzioni settecentesche, nel momento in cui si ha il riconoscimento del rapporto conflittuale tra Stato e società civile. Tale rapporto ci permette di definire anche le scansioni interne in termini di secoli: il XIX (dal 1776-89 al 1879), il XX (1880-1979), il XXI iniziato nel 1980.
\end{abstract}

\section{Il concetto di contemporaneità}

La contemporaneità è una categoria sfuggente, di competenza dei filosofi, come ricordava tempo fa Mariuccia Salvati. Agli storici spetta la riflessione sull'età contemporanea, vale a dire su come individuare scansioni temporali utili a organizzare lo studio del passato.

Prima di entrare nel merito dobbiamo però chiederci come mai questo tema è sempre più presente nel dibattito pubblico. Credo si possa dire che oggi in Italia interrogarsi sul significato dell'età contemporanea rappresenti il precipitato di una tensione intellettuale e di una inquietudine sociale che spinge gli storici a cercare di «occupare» il presente con gli strumenti dell'indagine storica. Gli storici, sempre più emarginati nella sfera del dibattito pubblico, vengono spesso sollecitati a misurarsi con temi un tempo ritenuti di pertinenza della cronaca. Siamo nell'epoca della «presentificazione» come mai prima d'ora e gli storici non possono non esserne influenzati. Quando tutto viene schiacciato sul presente, non solo si tende a perdere la memoria pubblica, peraltro destinata ad assottigliarsi sempre più sotto il peso dell'accelerazione delle informazioni e 
dell'estendersi dei canali di comunicazione, ma si cerca di irrompere nell'attualità, dando veste «scientifica» a riflessioni su vicende appena trascorse e su protagonisti ancora sulla scena pubblica.

\section{La cesura fra storia contemporanea e cronaca}

La cronaca, il presente, i current affairs dovrebbero però rimanere tali sino a quando, come diceva Geoffrey Barraclough, i problemi sollevati non finiscono per assumere una chiara fisionomia. Ed è qui il punto. Come si stabilisce la chiara fisionomia? Il tempo trascorso è il criterio più ovvio, ma spesso è anche la fine certa di un fenomeno o di un periodo a convincere gli storici ad avviare le loro indagini. Benedetto Croce si è arrestato al 1915 nella storia d'Italia scritta nel 1927 perché quell'anno indicava un confine esplicito, quello dell'Italia in tempo di pace; così come il 1945 ha favorito l'immediata proliferazione di storie sul ventennio fascista in quanto esisteva una conclusione inoppugnabile: la caduta del regime di Mussolini. Dove non è identificabile una cesura netta ed evidente risulta ancora più difficile districarsi tra storia e cronaca, tra documenti e memoria, anche se non solo nulla osta, ma è anzi auspicabile che si possano studiare eventi appena trascorsi con metodi d'indagine seri e rigorosi. Credo tuttavia sia inutile, se non controproducente, cercare la contemporaneità inseguendo il tempo presente o, nel migliore dei casi, il passato prossimo. La contemporaneità si dovrebbe definire analizzando le costanti, le categorie di fondo invariate che ci legano al passato al di là degli aspetti effimeri di cambiamenti di forma e non di sostanza. Ovviamente definire tali costanti fa parte dell'interpretazione dello studioso ed è per questo che la categoria della contemporaneità rappresenta per gli storici una sfida complessa, in quanto li costringe a individuare un «big-bang» dentro cui ci troviamo ancora immersi.

\section{Il «big-bang» della storia politica e sociale}

Nell'ambito della storia politica e sociale, a mio avviso, quel «big-bang» è rappresentato dalle Rivoluzioni americana e francese. Le porte del mondo contemporaneo, per l'Europa e le sue occidentali «diramazioni» transoceaniche, si sono aperte con le rivoluzioni settecentesche. Un «bigbang» che non ha ancora esaurito la sua carica propulsiva e ha dato origine ai cardini della modernità politica a cominciare dagli Stati-nazione, con tutto quello che ne consegue in termini di legittimazione popolare, vale a dire il nuovo, insostituibile, cemento del potere pubblico. Da questo punto di vista siamo, dunque, oggi ancora contemporanei ai nostri avi di fine ' 700 , ci troviamo cioè nello stesso tempo storico iniziato allora. Tuttavia, all'interno di tale gigantesca onda d'urto, che potremmo definire quella del costituzionalismo rappresentativo, si possono individuare profonde differenze, corrispondenti a precise scansioni temporali e tutte definibili in termini di variazioni all'interno del nuovo rapporto Stato-società forgiato dalle rivoluzioni costituzionali e liberali settecentesche. Se assumiamo la prospettiva della dinamica politica profonda che scaturisce da quel decisivo rapporto, possiamo assumere che il XIX secolo sia il periodo che ha origine dalla Rivoluzione francese del 1789 e arriva agli anni Ottanta dell'Ottocento, mentre il XX copre i cento anni - un secolo esatto - che vanno da allora fino agli anni Ottanta del Novecento. 


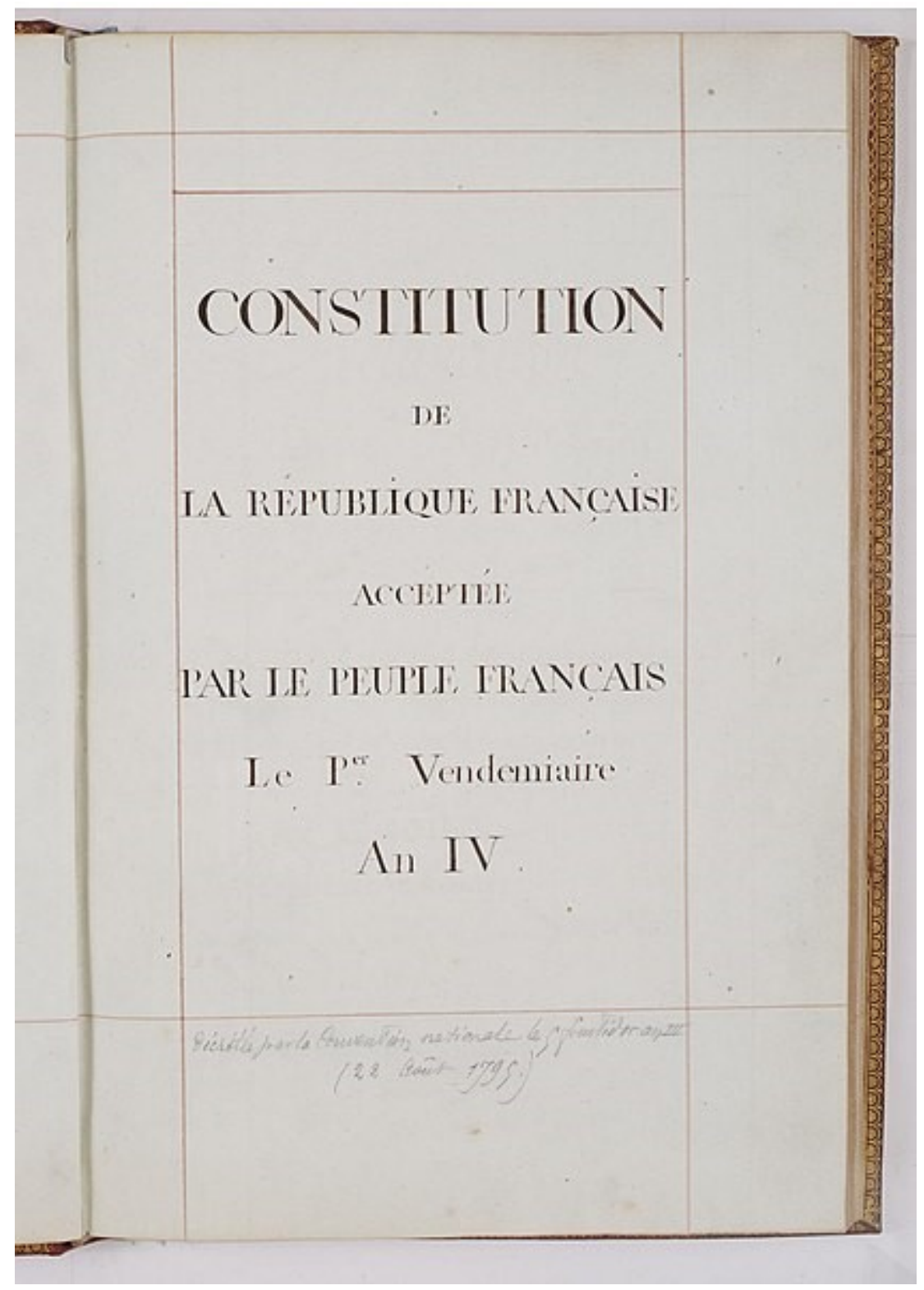

La bozza della Costituzione francese del 1795 (Archives nationales)

\section{Le Costituzioni fra Stato e individuo}

La contemporaneità si apre dunque con il XIX secolo, vale a dire con il ridimensionamento del ruolo dello Stato e l'emersione dell'individuo, del soggetto politico sulla scena pubblica. La Costituzione era posta a fondamento dell'organizzazione dei sistemi politici a dimostrazione che non era più possibile detenere il potere senza legittimazione pubblica e rispetto dei diritti «dell'uomo e del cittadino». L'individuo, ora tutelato dal potere politico, iniziava così il lungo percorso della contemporaneità in cui si caratterizzava come soggetto attivo del processo di formazione della decisione politica. Nel rapporto tra Stato e Società il sovrano perdeva il carattere assoluto e poteva conservare un ruolo attivo solo in presenza di un patto costituzionale che lo legava all'unico corpo politico in grado di garantire la legittimazione del sistema: la nazione. Nel XIX secolo lo Stato si fa nazione (e viceversa) e il liberalismo s'impone come l'orizzonte ideologico in grado d'interpretare la nuova logica economica e sociale. Siamo di fronte a una mutazione che farà degli Stati non più le sedi del potere assoluto, bensì il luogo di comunità di partecipazione politica, più o meno mitiche e culturalmente omogenee. È l'ambiente in cui gli individui, imponendo la Costituzione ai sovrani, prendono consapevolezza di un protagonismo politico che li affranca dal 
ruolo di sudditi e li proietta in quello di cittadini. L'immaginario del XIX secolo è composto da questi soggetti giuridicamente liberi, la cui azione mette in crisi gli antichi bastioni familiari e di ceto per fare dell'individuo la misura di ogni cosa.

\section{Stato-nazione e società}

Sono però gli anni Ottanta dell'Ottocento a rappresentare il simbolico spartiacque tra XIX e XX secolo. Il congresso di Berlino del 1878 aveva di fatto - dietro l'apparenza del congelamento dello status quo imposto dalla pax germanica - gettato le basi per un'intensificazione delle rivalità tra gli Stati che avrebbe ben presto dato i suoi frutti, a cominciare dalla perenne eccitazione di opinioni pubbliche sempre più portate a considerare lo Stato uno strumento demandato a competere sulla scena internazionale in termini di agonismo nazionalista. Chi intende individuare una data simbolica per definire l'ingresso nel XX secolo deve guardare al luglio del 1879, quando il Parlamento tedesco avallò la decisione bismarckiana di mettere in movimento la slavina protezionista che negli anni successivi avrebbe travolto il principale pilastro della cultura liberale, la libertà dei commerci. Si è trattato di una scelta foriera di un nuovo modo d'intendere la politica economica, che (almeno virtualmente) dagli individui tornava nelle mani degli Stati impegnati a ridisegnare, in funzione di criteri di contenimento sociale, i bilanci pubblici con la stessa logica dei rapporti diplomatici, rendendoli cioè più attenti alle sirene del riarmo, della militarizzazione e delle rivalità tra nazioni.

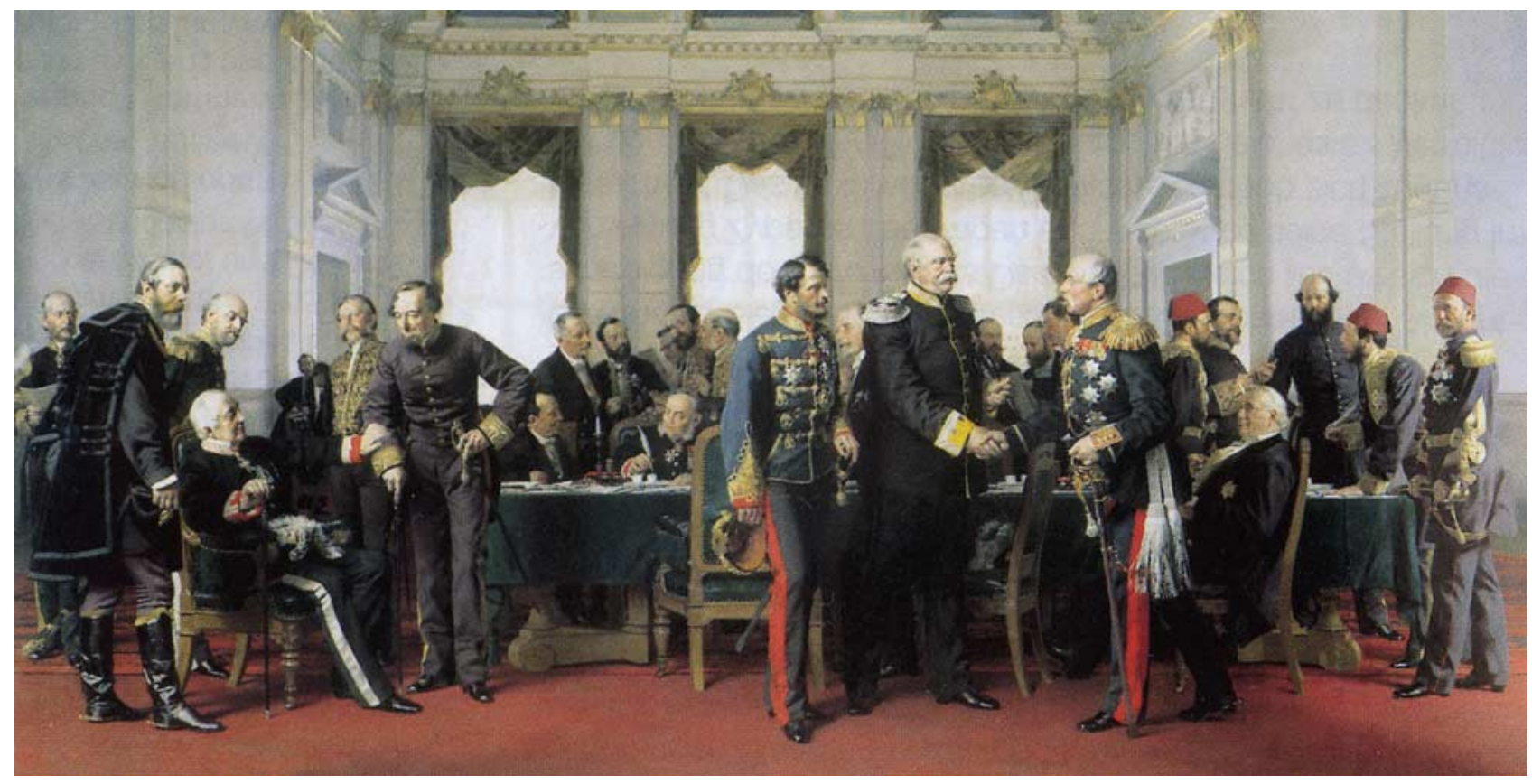

Il Congresso di Berlino (A. von Werner, 1881)

L'ingresso nel XX secolo, sempre all'interno dell'onda lunga della contemporaneità, vide dunque lo Stato-nazione trasformarsi in strumento per assoggettare la società. Si diffuse una nuova cultura politica che vide il declino del soggetto individuale e la crescita di nuove sensibilità collettive, dai sindacati ai partiti, dalle associazioni ai movimenti che hanno nello Stato un punto di riferimento ineludibile per quanto polemico e conflittuale. Un'epoca era finita e ne stava per cominciare un'altra. Lo registrò con grande efficacia Antonio Labriola nel 1890: «Persuadiamoci che con il glorioso centenario del 1889 è ormai chiuso il periodo della rivoluzione liberale, cominciato il 20 aprile del 1789. L'individualismo cede il posto alla socialità»»[1]. 


\section{Il centralismo statalista}

Quello che un tempo era lo Stato assoluto, intrusivo, da colpire perché nemico del nuovo soggetto storico, il cittadino, ormai cominciava ad essere percepito come un potenziale strumento di protezione indispensabile, attraverso le sue articolazioni - ministeri, apparati burocratici, enti, agenzie amministrative - , nel fronteggiare, da un lato, un capitalismo sempre più manageriale e, dall'altro, i movimenti di classe. Da questo punto di vista non sembra rappresentare uno spartiacque neppure l'instaurazione dei regimi fascista e nazista, che si presentano come risposta patologica e totalitaria al problema del conflitto Stato-società, interpretato come frattura di una mitica ed organica entità unitaria da ricomporre a ogni costo, eliminando ogni fonte di conflitto sociale. La centralità che da un lato all'altro dell'Atlantico assunse lo Stato pianificatore e organizzatore sembra quantomeno attenuare distanze politiche altrimenti siderali tra la democrazia avanzata di Roosevelt e i regimi totalitari europei.

\section{Gli esordi del XXI secolo}

Le due guerre mondiali hanno finito per rafforzare l'idea della centralità dello Stato, visto che l'intervento pubblico in quei drammatici frangenti divenne parte rilevante della vita quotidiana. Così, archiviata l'età dei totalitarismi, il trentennio compreso tra la fine della Seconda guerra mondiale e gli anni Ottanta non mostra ripensamenti dal punto di vista dell'intervento pubblico gestito dallo Stato-nazione. Ora, ovviamente, le democrazie liberali lo declinano attraverso il riconoscimento del pluralismo dei partiti e dei sindacati, legittimati dalla Resistenza alle dittature e ormai diventati espressione semi-istituzionale della volontà della società civile.

Perché nel 1980, proprio duecento anni dopo l'esordio della contemporaneità, ha fine il XX secolo e inizio il XXI secolo? Perché forse il difficile rapporto tra Stato e società che aveva attraversato i due secoli precedenti, con la prevalenza ora dell'uno ora dell'altra, aveva subito un altro radicale mutamento e cominciava a risolversi in un paradossale indebolimento di entrambi. Insomma, se lo Stato piange, non è che la società rida. La società civile - intesa come insieme di associazioni, partiti, sindacati, cittadinanza organizzata, ideologie - esiste e cresce in funzione della presenza di uno Stato forte. La sua crisi, iniziata allora, oggi riflette la minore compattezza sociale, frutto anche di una rivoluzione tecnologica che crea reti senza socializzazione, e l'indebolirsi della sovranità statale, a partire dalle sfide, appena iniziate, all'integrità territoriale nazionale. Una sovranità messa in discussione dalla globale e incontrollata forza del capitalismo finanziario, che però, se talvolta riduce l'autonomia dei governi, non ne sta intaccando la dimensione e la volontà pedagogica.

\section{La crisi dello Stato e della società fra vecchie e nuove categorie interpretative}

Il contemporaneo e paradossale indebolimento dello Stato e della società è sintomo, e allo stesso tempo implica, la crisi profonda delle categorie che avevano segnato la modernità politica cittadinanza, diritti, sovranità - non più capaci di costituire i fondamenti dell'ordine politico, perché essi stessi soggetti a contraddizioni, ridefinizioni e fratture. Divisione, separazione, polarizzazione, frantumazione sembrano invece costituire lo sfondo concettuale entro cui tutte le master narratives, che avevano contribuito a dare forma e significato all'ordine della modernità, si stanno disintegrando. 


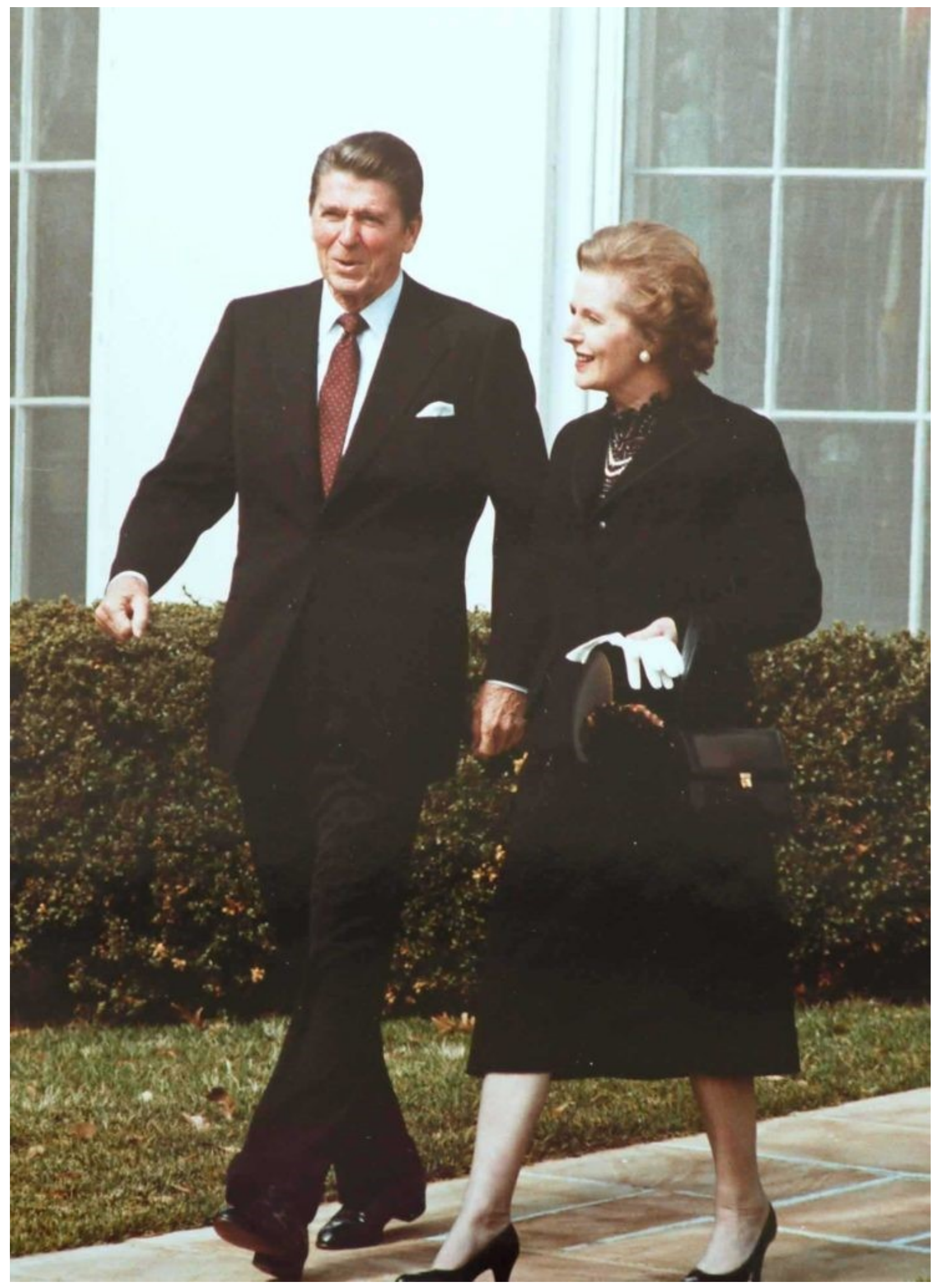

Ronald Reagan e Margaret Thatcher, febbraio 1981 
Il XXI secolo continua per alcuni versi a rimanere fermo al suo esordio, vale a dire alla lotta, avviata da Thatcher e Reagan e oggi rivendicata da Donald Trump, per la demolizione dello Stato amministrativo. Per altri versi, però, si presenta come un'inedita frammentazione del rapporto individuo-Stato-società, amplificata dal disorientamento causato dagli intensi fenomeni migratori in corso, che ha i suoi effetti più evidenti nella scomparsa della credibilità politica dei parlamenti $\mathrm{e}$ quindi nella crisi della rappresentanza. Con il declino dello Stato-regolatore, ciò che appare con chiarezza è soprattutto lo squilibrio tra ricchezza e democrazia. Non sappiamo quanto durerà, dal punto di vista politico, questo nuovo secolo, né dove ci porterà, però dovremmo sempre ricordarci, come diceva John Maynard Keynes, che l'inevitabile non accade mai, l'inatteso sempre.

\section{Bibliografia}

- F. Cammarano, Il XX secolo, in R. Brizzi, F. Cammarano, S. Cavazza, G. Guazzaloca, M. Marchi (a cura di), Fare Storia politica. Studi dedicati a Paolo Pombeni, Viella, Roma 2018

- C. Pavone (a cura di), '900. I tempi della storia, Donzelli, Roma 1997

- G. Galasso, Nient'altro che storia. Saggi di teoria e metodologia della storia, Il Mulino, Bologna 2000

- R. Vivarelli, I caratteri dell'età contemporanea, Il Mulino, Bologna 2005

Note:

[1] A. Labriola, Lettera ad Alfredo Baccarini, in Id., Scritti Politici, a cura di V. Gerratana, Bari, Laterza, 1970, pp. 202-3. 